\title{
CD19-targeted CAR regulatory T cells suppress B cell pathology without GvHD
}

\author{
Yuki Imura, ${ }^{1,2}$ Makoto Ando, ${ }^{1}$ Taisuke Kondo, ${ }^{1,3}$ Minako Ito, ${ }^{4}$ and Akihiko Yoshimura ${ }^{1}$ \\ 'Department of Microbiology and Immunology, Keio University School of Medicine, Tokyo, Japan. ${ }^{2}$ Sohyaku Innovative \\ Research Division, Mitsubishi Tanabe Pharma Corp., Yokohama, Japan. ${ }^{3}$ Pediatric Oncology Branch, National Cancer \\ Institute, NIH, Bethesda, Maryland, USA. ${ }^{4}$ Medical Institute of Bioregulation, Kyushu University, Fukuoka, Japan.
}

Regulatory T cells (Tregs) play essential roles in maintaining immunological self-tolerance and preventing autoimmunity. The adoptive transfer of antigen-specific Tregs has been expected to be a potent therapeutic method for autoimmune diseases, severe allergy, and rejection in organ transplantation. However, effective Treg therapy has not yet been established because of the difficulty in preparing a limited number of antigen-specific Tregs. Chimeric antigen receptor (CAR) T cells have been shown to be a powerful therapeutic method for treating B cell lymphomas, but application of CAR to Treg-mediated therapy has not yet been established. Here, we generated CD19-targeted CAR (CD19-CAR) Tregs from human PBMCs (hPBMCs) and optimized the fraction of the Treg source as $C D 4^{+} \mathrm{CD} 25^{+} \mathrm{CD} 127^{\circ} \mathrm{CD} 45 R \mathrm{C}^{+} \mathrm{CD} 45 \mathrm{RO}-{ }^{-}$. CD19-CAR Tregs could be expanded in vitro while maintaining Treg properties, including high expression of the latent form of TCF- $\beta$. CD19-CAR Tregs suppressed IgC antibody production and differentiation of B cells via a TCF- $\beta$-dependent mechanism. Unlike conventional CD19-CAR CD8 ${ }^{+}$T cells, CD19-CAR Tregs suppressed antibody production in immunodeficient mice that were reconstituted with hPBMCs, reducing the risk of graft-versus-host disease. Therefore, the adoptive transfer of CD19-CAR Tregs may provide a novel therapeutic method for treating autoantibody-mediated autoimmune diseases.

Conflict of interest: The authors have declared that no conflict of interest exists.

Copyright: () 2020, American Society for Clinical Investigation.

Submitted: January 7, 2020

Accepted: June 4, 2020

Published: July 23, 2020.

Reference information: /CI Insight. 2020;5(14):e136185.

https://doi.org/10.1172/jici.

insight.136185.

\section{Introduction}

Tregs suppress excess immunity against a variety of antigens, including self-antigens, commensal bacteria-derived antigens, and environmental allergens. Thus, Tregs have been shown to play important roles in suppressing the development of autoimmunity, colitis, and allergies (1). In humans, some clinical trials of adoptive transfer of ex vivo expanded polyclonal Tregs have been performed to suppress graft-versus-host disease (GvHD) after allogeneic hematopoietic stem cell transplantation (2-5) and type 1 diabetes (T1D) (6-8). These phase I studies revealed that the adoptive transfer of polyclonal Tregs is well tolerated and showed promising effects. Although the results of polyclonal Treg adoptive therapy are encouraging, a large number of cells are necessary, and the risk of nonspecific immunosuppression should be considered.

The adoptive transfer of antigen-specific Tregs was shown to be a more promising approach because fewer cells are needed to suppress targeted cells, which reduces the risk of nonspecific immunosuppression $(9,10)$. There are several strategies to generate antigen-specific Tregs, which overexpress a transgenic $\mathrm{T}$ cell receptor (TCR) or expand when stimulated with antigen. These antigen-specific Tregs indicate superior suppression of nonspecific or polyclonal Tregs for several diseases, such as GvHD (11), hemophilia A (12), and experimental autoimmune encephalomyelitis (EAE) (13). In mice, in vitro generation of induced antigen-specific Tregs (iTregs) in the presence of vitamin C, which stabilizes Foxp3 expression, has been used to suppress GvHD (14), but methods for generating stabilized human iTregs have not yet been established (15).

Another way to create antigen-specific $\mathrm{T}$ cells is through chimeric antigen receptor (CAR) expression, which consists of an extracellular single chain antibody ( $\mathrm{scFv}$ ) fused to intracellular signaling domains in primary $\mathrm{T}$ cells. These CAR T cells have been successfully used in cancer treatment and are approved for treatment of patients with certain types of B cell lymphoma $(16,17)$. Many clinical trials are ongoing in multiple cancers using adoptive transfer of CAR T cells that recognize multiple tumor antigens (18). CAR therapy is a way to facilitate interaction of Tregs with target cells. CAR Tregs may be safer than conventional CAR T cells because of the low inflammatory effects and cytotoxicity of Tregs. Some studies have 
shown that adoptive transfer of CAR Tregs suppresses autoimmunity against GvHD (19), hemophilia A (20), EAE (21), and colitis (22) at the preclinical level.

Autoantibodies secreted from B cells are thought to induce various autoimmune diseases (23), such as systemic lupus erythematosus (SLE) (24), Sjögren's syndrome (25), and IgG4-related diseases (26). B cell-targeting therapy, such as anti-BAFF neutralizing antibodies (belimumab) or anti-CD20 depleting antibodies (rituximab), are used to treat severe autoantibody-mediated autoimmune diseases (27). Although one report used conventional CD19-targeted CAR (CD19-CAR) CD8 ${ }^{+} \mathrm{T}$ cells for the treatment of SLE in murine SLE models (28), there are no reports on the application of CD19-CAR Tregs for autoimmune B cell control. In this study, we demonstrated that CD19-CAR Tregs suppress the antibody production of B cells without harmful side effects such as GvHD. The adoptive transfer of CD19-CAR Tregs might provide a novel therapeutic method to treat autoantibody-mediated autoimmune diseases.

\section{Results}

Experimental design to create CD19-targeted CAR Tregs from human peripheral blood T cells. We mainly used the CD19-targeted second generation CAR (CD19-CAR), which consists of the FMC63 extracellular single-chain variable fragment ( $\mathrm{scFv}$ ) domain fused to the $\mathrm{CD} 28$ hinge, transmembrane, and cytoplasmic domains and the CD3 $\zeta$ cytoplasmic domain (1928z); the Venus gene is expressed via internal ribosome entry site-2 (IRES2) to detect gene transduction (Figure 1A) (29). Human Tregs $\left(C D 4^{+} C D 25^{+} C D 127^{10}\right.$ ) are classified into 3 main subsets based on Foxp3, CD25, and CD45RA expression levels, as follows: CD45RA ${ }^{+}$ Foxp $^{\text {lo }} / \mathrm{CD} 25^{\text {lo }}$ naive/resting Tregs; CD45RA-Foxp3 $3^{\text {hi }} / C D 25^{\text {hi }}$ effector Tregs; and CD45RA-Foxp3 $3^{\text {lo }} /$ $\mathrm{CD}_{25} 5^{\text {lo }}$, mainly consisting of non-Tregs $(30,31)$. The naive/resting Treg fraction was first enriched from healthy donor PBMCs by magnetic negative selection and then purified by FACS based on marker expression, with $\mathrm{CD}^{+} \mathrm{CD}^{2} 5^{+} \mathrm{CD} 127^{\mathrm{lo}} \mathrm{CD} 45 \mathrm{RA}^{+} \mathrm{CD} 45 \mathrm{RO}^{-}$cells, which were considered to be naive/resting Tregs (CD45RA ${ }^{+}$Tregs) (Figure 1B). We also sorted $\mathrm{CD}^{+} \mathrm{CD}^{+} 5^{+} \mathrm{CD} 127^{10} \mathrm{CD} 45 \mathrm{RA} \mathrm{CD}^{-} \mathrm{C} 5 \mathrm{RO}^{+} \mathrm{T}$ cells, which were considered to be effector/non-Tregs (CD45RO ${ }^{+}$Tregs), and CD4 ${ }^{+} \mathrm{CD} 25^{-} \mathrm{CD} 127^{+} \mathrm{CD} 45 \mathrm{RA}^{+} \mathrm{CD} 45 \mathrm{RO}^{-}$ $\mathrm{T}$ cells, which represented conventional $\mathrm{T}$ cells (Tconvs) (Figure 1B). CD19-CAR retroviruses were transduced into these 3 fractions, and then Venus ${ }^{+}$cells were sorted and expanded for 8 days by coculturing with human CD19-expressing K562 (hCD19-K562) cells in the presence of IL-2 (Figure 1C), while empty virus-infected (polyclonal) Tregs were expanded by stimulation with anti-CD3/CD28 beads with IL-2. The CD19-targeted CAR transduction rate in CD45RA ${ }^{+}$Tregs (CD19-CAR CD45RA ${ }^{+}$Tregs) and Tconvs (CD19-CAR Tconvs) was approximately $50 \%$ and $20 \%$, respectively (Figure 1D). The CD19-CAR transduction rate in $\mathrm{CD}_{4} \mathrm{RO}^{+}$Tregs (CD19-CAR CD45RO ${ }^{+}$Tregs) was around 50\% (data not shown). The number of CD19-CAR CD45RA+ Tregs increased approximately 50- to 80-fold 8 days after the start of expansion, which was comparable to the proliferation of polyclonal Tregs (Figure 1E) but slightly lower than that of CD19-CAR Tconvs.

Optimization of CD19-targeted CAR Treg induction. Both CD45RA ${ }^{+}$and CD45RO ${ }^{+}$Tregs expressed high levels of Foxp3 and Helios immediately after isolation (Figure 2A). However, after CAR transduction and expansion, CD19-CAR CD45RA ${ }^{+}$Tregs and polyclonal Tregs retained high levels of Foxp3, Helios, and CTLA-4, while CD19-CAR CD45RO ${ }^{+}$Tregs lost most of this expression (Figure $2 \mathrm{~B}$ and Supplemental Figure 1A; supplemental material available online with this article; https://doi.org/10.1172/jci. insight.136185DS1). CD19-CAR CD45RA ${ }^{+}$Tregs produced high levels of the antiinflammatory cytokine IL-10, which was comparable to CD19-CAR CD45RO ${ }^{+}$Tregs, while they expressed much less IFN- $\gamma$ and IL-2 compared with CD19-CAR CD45RO+ Tregs and CD19-CAR Tconvs (Figure 2C and Supplemental Figure 1, B-D; data for Tconvs are shown in Supplemental Figure 2, A-C). TGF- $\beta$ has been shown to be involved in Treg suppression functions (32), which are usually associated with latency-associated peptide (LAP), and is also known as latent TGF- $\beta$. It is activated by glycoprotein A repetitions predominant protein (GARP) (33) or other membrane proteins such as integrins and proteases (32). Surface LAP and GARP were significantly increased in CD19-CAR CD45RA ${ }^{+}$Tregs after coculture with hCD19-K562 cells but not with parental K562 cells (Figure 2D), and the percentage of $\mathrm{LAP}^{+} \mathrm{GARP}^{+}$cells was much higher compared with that in CD19-CAR CD45RO ${ }^{+}$Tregs or CD19-CAR Tconvs (Figure 2D; data for Tconvs are shown in Supplemental Figure 2D). Next, we examined the cytotoxicity of CD19-CAR Tregs against CD19+ ${ }^{+}$ CD19- K562 cells. To distinguish the cytotoxic effects and suppression of proliferation, we used irradiated K562 cells because K562 cells doubled within 24 hours. CD19-CAR Tconvs killed CD19-expressing K562 cells in an antigen-specific manner, while CD19-CAR Tregs did not (Figure 2E and Supplemental Figure 3A). 
A

\begin{tabular}{|c|c|c|c|c|c|}
\hline & & & IRES2 & Venus & Fmnty \\
\hline $\begin{array}{l}\text { FMC63 scFv } \\
\text { (anti-hCD19) }\end{array}$ & $\begin{array}{c}\text { CD28 } \\
\text { (EC-TM-CP) }\end{array}$ & $\begin{array}{l}\text { CD3Z } \\
\text { (CP) }\end{array}$ & IRES2 & Venus & $\begin{array}{l}\text { CD19-targeted } \\
\text { CAR (1928z) }\end{array}$ \\
\hline
\end{tabular}

B

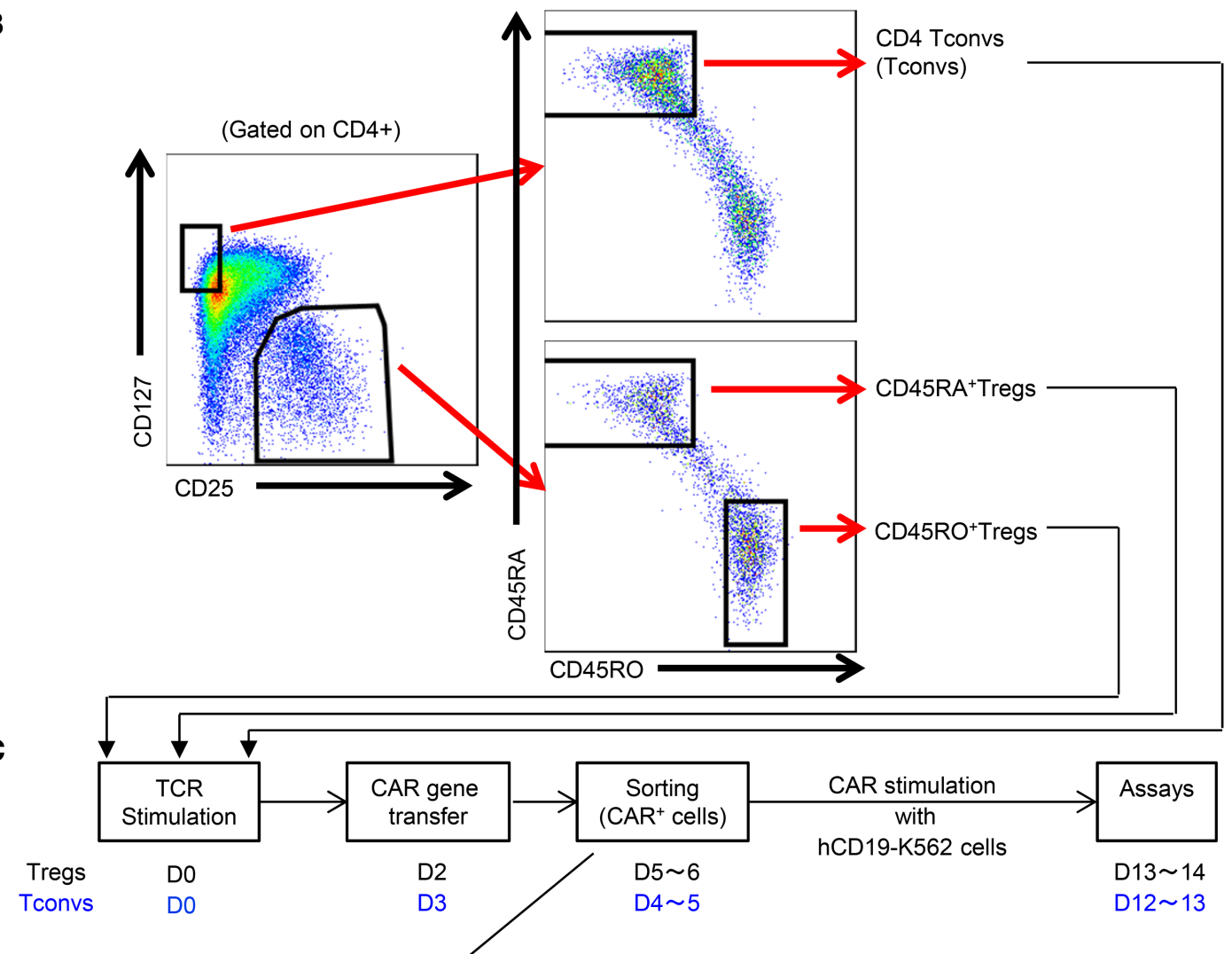

C

D

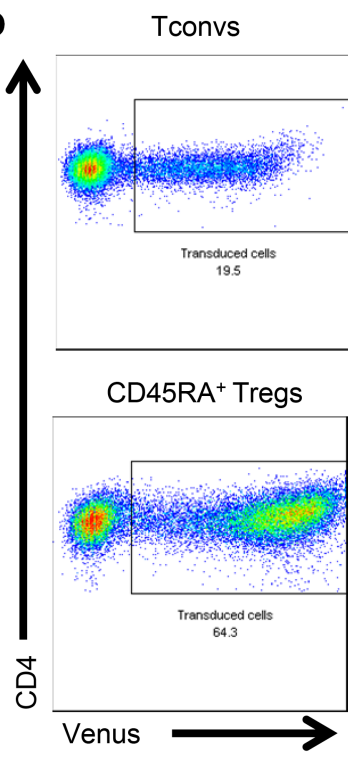

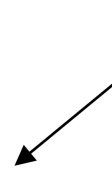

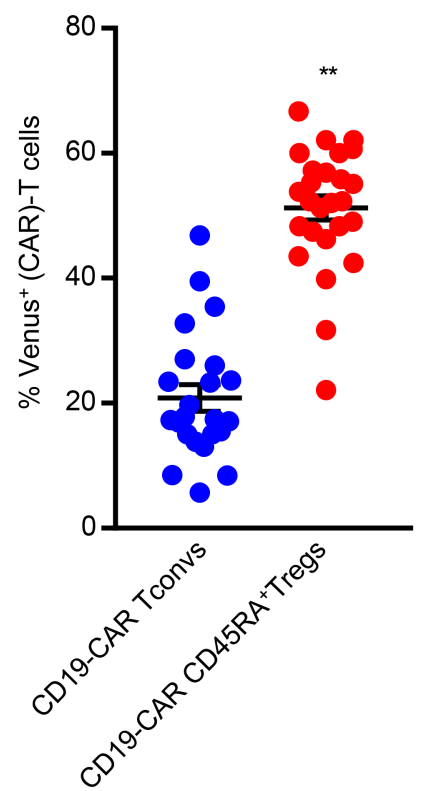

E

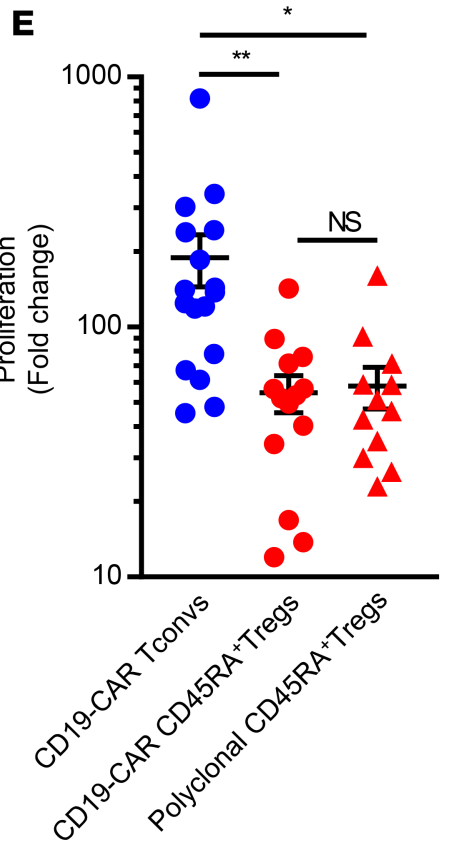


Figure 1. Generation of CD19-targeted CAR Tregs. (A) Schematic representation of constructs carrying CD19-targeted CAR and empty vector. EC, extracellular domain; TM, transmembrane domain; CP, cytoplasmic domain. (B) Sorting strategy to isolate naive/resting CD45RA+ regulatory T cells (CD45RA+ Tregs), CD45RO+ Tregs, and conventional CD4 T cells (Tconvs). (C) Scheme for the generation and expansion of CD19-targeted CAR (CD19-CAR) Tregs and CD19-CAR Tconvs. (D) Flow cytometric analysis of CAR (Venus) when transduced cells were sorted on days 4-6. The left panels show representative FACS profiles, and the right panel shows quantification of the transduction rate $(n=23$ or 26). Data are representative of 26 independent experiments using human samples that were provided by 5 healthy donors. (E) Fold expansion on days 12-14, 8 days after CAR T cells were sorted ( $n=17,14$, or 12). Data are representative of 17 independent experiments using human samples provided by 5 healthy donors. $P$ values were determined using (D) 2 -tailed Student's $t$ test or (E) 1 -way ANOVA ( $\left.{ }^{*} P<0.05,{ }^{* *} P<0.01\right)$. Data are presented as mean \pm SEM.

These results were supported by the finding that CD19-CAR Tregs expressed lower levels of granzyme B and perforin compared with CD19-CAR Tconvs and CD19-CAR CD8 ${ }^{+} \mathrm{T}$ cells (Supplemental Figure 3B). Thus, these data suggest that unlike conventional CAR T cells, CAR Tregs have little cytotoxic activity.

On the basis of these data, we conclude that CD45RA+ Tregs are suitable for CD19-targeted CAR Treg generation.

To optimize the costimulatory domain of CD19-CAR, we compared CD28-based CD19-targeted CAR (1928z) and 4-1BB-based CD19-targeted CAR (19BBz). Foxp3, Helios, and CTLA-4 expression levels in 19BBz Tregs were slightly lower than those in 1928z Tregs (Figure 3A). 1928z Tregs proliferated in response to target cells faster than 19BBz Tregs did (Figure 3B). Furthermore, IL-10 expression in 1928z Tregs was higher than that in 19BBz Tregs (Figure 3C and Supplemental Figure 4). In addition, 1928z Tregs expressed higher levels of LAP/GARP than 19BBz Tregs (Figure 3D). Consistently, TGF- $\beta 1$ was secreted in supernatants from CD19-CAR Tregs in response to target CD19-K562 cells, and TGF- $\beta 1$ levels were higher in 1928z Tregs compared with 19BBz Tregs (Figure 3E). Therefore, we considered that CD28based CD19-CAR is more effective than 4-1BB-based CD19-CAR for CAR Treg generation; hereafter, we refer to CD28-based CD19-targeted CAR CD45+ Treg as CD19-CAR Tregs.

CD19-targeted CAR Tregs suppress antibody production from primary B cells in vitro. Next, we studied the effect of CD19-CAR Tregs on the proliferation and antibody production of primary human B cells in vitro. Primary B cells were isolated by magnetic-activated cell sorting (MACS) and stimulated with anti-IgM and anti-CD40 antibodies in the presence of IL-21, which induced extensive proliferation, class-switch recombination, and plasma cell differentiation (34). CD19-CAR Tregs suppressed proliferation of B cells more efficiently than empty vector-transduced Tregs (polyclonal Tregs) (Figure 4A). In contrast, CD19CAR Tregs suppressed proliferation of conventional $\mathrm{T}$ cells as efficiently as polyclonal Tregs, suggesting that CD19-CAR Tregs retain the suppressive activity of empty vector-treated Tregs (Supplemental Figure 5A). CD19-CAR Tregs also strongly suppressed IgG production from primary B cell differentiation (Figure $4 \mathrm{~B}$ and Supplemental Figure $5 \mathrm{~B}$ ). TGF- $\beta$ was reported to induce IgA class switching and reduce IgG production $(35,36)$. Because CD19-CAR Tregs expressed high levels of TGF- $\beta$ (see Figure $2 \mathrm{D}$ and Figure $3 \mathrm{E})$, there is a possibility that IgA class switching is promoted by CD19-CAR Tregs. However, CD19-CAR Tregs suppressed both IgG and IgA production (Figure 4C).

It has been reported that CAR expression may produce tonic signals in the absence of antigen binding (37). To examine whether CAR expression itself affects CAR Treg functions, we generated HER2-targeted CAR Tregs (HER2-CAR Tregs) (38) and compared them with CD19-CAR Tregs. Similar to polyclonal Tregs, HER2-CAR Tregs suppressed neither proliferation of B cells nor antibody production (Supplemental Figure 5C).

CD19-CAR Tregs suppress B cells through a TGF- $\beta$-dependent mechanism. Next, to investigate the mechanism whereby CD19-CAR Tregs suppressed B cells, we tested various antibodies against known effectors of Tregs, including TGF- $\beta$, IL-10, CTLA-4, PDL-1, and FasL (Figure 5A, 1eft). Among them, anti-TGF- $\beta$ neutralizing antibody partly, but significantly, reverted the suppressive effect of CD19-CAR Tregs on IgG production in B cells. This effect was confirmed using CA19-CAR Tregs from a different donor (Figure $5 \mathrm{~A}$, right). Furthermore, a TGF- $\beta$ type 1 receptor kinase inhibitor, RepSox, almost completely reverted the suppressive effect of CD19-CAR Tregs at $1 \mu \mathrm{M}$ (Figure 5, B and C).

Since full TGF- $\beta$ production required stimulation of CD19-CAR Tregs by target cells (Figure 2D and Figure 3E), interaction between CD19-CAR Tregs and B cells may be necessary. As expected, Transwell culture preventing cell-cell contact canceled the suppression of B cell proliferation by CD19-CAR Tregs (Supplemental Figure 5D).

Since some reports have shown that TGF- $\beta$ suppresses IgG production in B cells in vitro (39), these data strongly suggest that CD19-CAR-mediated TGF- $\beta$ signals suppress the proliferation and IgG production in B cells without promoting IgA class switching. 
A

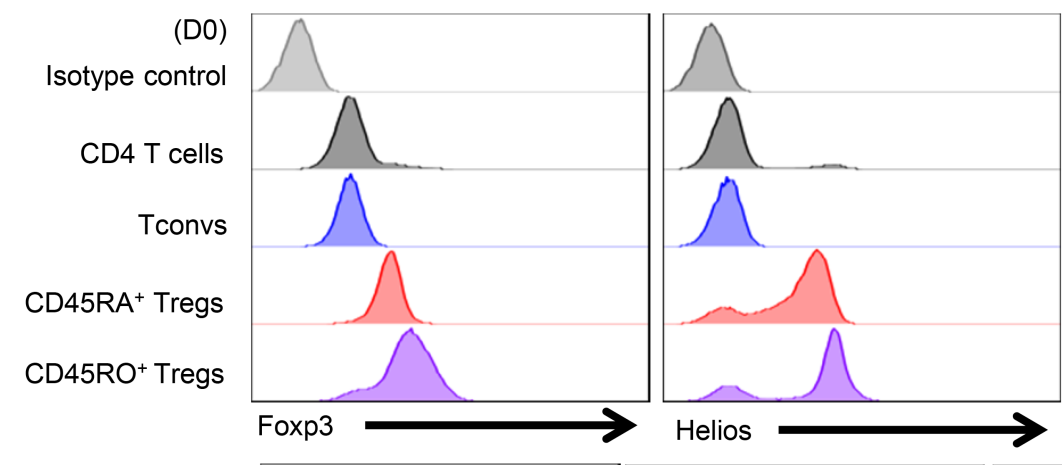

B (D13: after culture) Isotype control CD19-CAR Tconvs

Polyclonal Tregs CD19-CAR CD45RA+ Tregs CD19-CAR CD45RO+ Tregs
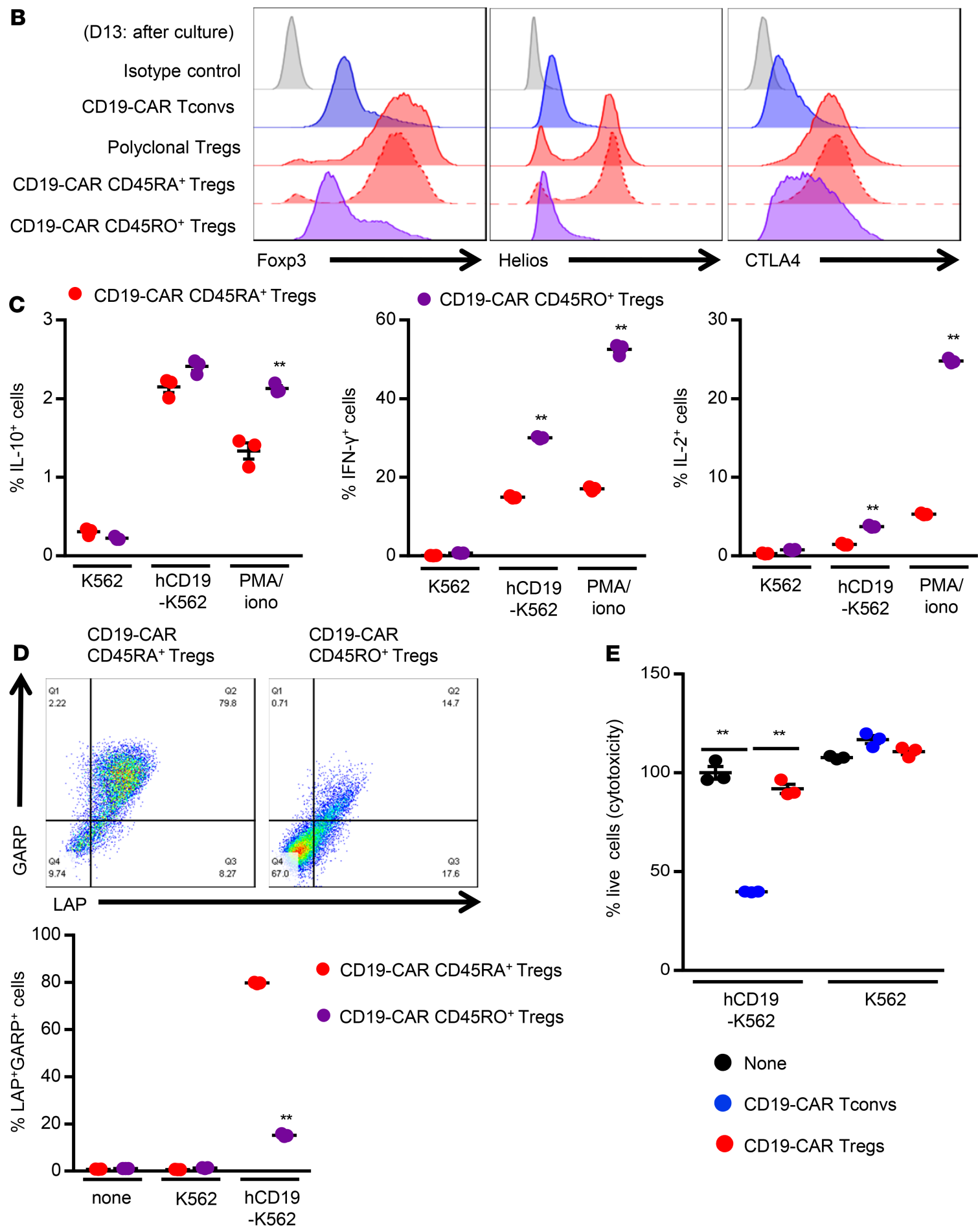

E

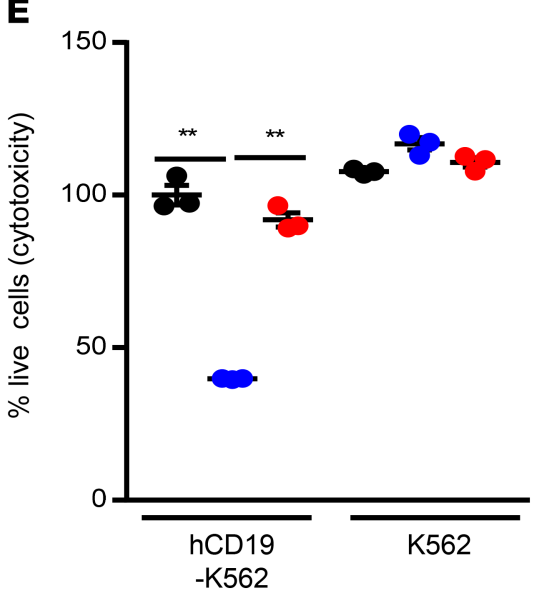

None

CD19-CAR Tconvs

CD19-CAR Tregs 
Figure 2. CD19-targeted CAR Tregs maintain the phenotype and suppressive abilities of Tregs after expansion. (A and B) Flow cytometric analysis of Foxp3, Helios, and CTLA-4 on day 0 after sorting (A) and day 13 after expansion (B). (C) Flow cytometric analysis of IL-10, IFN- $\gamma$, and IL-2 in the indicated cells 4 hours after stimulation with hCD19-K562 cells, K562 cells, or PMA/ionomycin (PMA/iono) $(n=3)$. (D) Flow cytometric analysis of LAP and GARP in the indicated cells 1 day after coculture with hCD19-K562 cells or K562 cells $(n=3)$. (E) Cytotoxicity of CD19-targeted CAR T cells 1 day after coculture with hCD19-K562 or K562 cells. The graph shows residual live targeted cells after coculture $(n=3)$. Data were collected using human samples provided by 1 healthy donor. $P$ values were determined using (C and $\mathbf{D}) 2$-tailed Student's $t$ test or $(\mathbf{E}) 1$-way ANOVA $\left({ }^{* *} P<0.01\right)$. Data are presented as mean \pm SEM.

CD19-targeted CAR Tregs suppress human B cells in vivo and suppress GvHD effects. Last, we investigated the suppressive function of CD19-CAR Tregs on B cells in vivo. Primary human PBMCs were adoptively transferred into immunodeficient NOD.Cg-Prkdscid $I l 2 r g^{\text {tm } l W j l} / \mathrm{Szj}$ (NSG) mice, which resulted in xenogeneic GvHD and human Ig production (40). When CD19-CAR Tregs were transferred into NSG mice 7 days after human PBMC transfer, both human IgG and IgM levels were reduced on days 14, 21, and 28 (Figure 6A). Polyclonal Tregs (empty vector-transduced Tregs) did not significantly reduce IgG and IgM levels on days 21 and 28 (Figure 6A). CD19-CAR Tregs, but not polyclonal Tregs, significantly suppressed CD20 $0^{+}$ cell expansion in peripheral blood from day 14 to day 28 (Figure 6B and Supplemental Figure 6A). Both transferred CD19-CAR Tregs and polyclonal Tregs were detected on day 14, and maintained similar levels 28 days after transfer (Figure 6C and Supplemental Figure 6B); the number of CD19-CAR Tregs was higher than the number of polyclonal Tregs at all time points. CD19-CAR Tregs also suppressed expansion of CD20-CD138+ plasma cells (Supplemental Figure 6C), indicating that CD19-CAR-Treg could suppress not only Ig production but also B cell differentiation to plasma cells. Similar data were obtained when CD19. CAR Tregs were simultaneously transferred with PBMCs (Supplemental Figure 7). These data indicate that CD19-CAR Tregs specifically suppress Ig production from B cells in vivo.

The transfer of human PBMCs resulted in severe GvHD, leading to weight loss and death, whereas the transfer of CD19-CAR Tregs alone did not result in any GvHD symptoms (Supplemental Figure 8A). Cotransfer of CD19-CAR Tregs suppressed GvHD resulting from PBMCs on day 28 (Figure 6D). This GvHD-suppressing effect of CD19-CAR Tregs was greater than that of polyclonal Tregs, probably because CD19-CAR Tregs, but not polyclonal Tregs, expanded in response to B cells. Since the transfer of human PBMCs in the absence of B cells also resulted in severe GvHD without detectable human IgG antibody (Supplemental Figure 8, B and C), B cells were not directly involved in GvHD effects. This suggests that the GVHD-suppressing effect of the CD19-CAR Tregs may be indirect; the exposure to CD19+ $\mathrm{B}$ cells in vivo activates CD19-CAR Tregs, which then suppress GVHD through their "usual" Treg TCRs.

A recent study showed that conventional CD19-CAR CD8 ${ }^{+} \mathrm{T}$ cells suppressed B cell-mediated autoimmune diseases by killing B cells (28). However, CD19-CAR CD8 ${ }^{+} \mathrm{T}$ cells may cause cytokine release syndrome $(41,42)$ (characterized by fever, hypotension, and respiratory insufficiency), which is associated with elevated serum cytokines, including IL-6. Thus, we compared the effects of CD19-CAR Tregs and CD19. CAR CD8 ${ }^{+} \mathrm{T}$ cells on GvHD in NSG mice reconstituted with human PBMCs. CD19-CAR Tregs or CD19. CAR CD8 ${ }^{+} \mathrm{T}$ cells were transferred 7 days after PBMC injection. Although CD19-CAR Tregs did not affect body weight by day 50 after transfer or reduce GvHD score, CD19-CAR CD8 ${ }^{+} \mathrm{T}$ cells promoted weight loss and did not reduce GvHD score (Figure 6E). Moreover, CD19-CAR CD8 ${ }^{+}$T cells, but not CD19-CAR Tregs, increased mouse IL-6 levels and decreased human Tregs 6 days after CD19-CAR CD8 ${ }^{+}$T cells or CD19-CAR Tregs were simultaneously transferred with PBMCs (Supplemental Figure 9). These data indicate that the transfer of CD19-CAR Tregs may be safer than that of CD19-CAR CD8 $8^{+} \mathrm{T}$ cells in autoimmune conditions.

\section{Discussion}

In this study, we optimized the conditions for generating stable CD19-targeted CAR Tregs, which are suitable for the adoptive cell therapy of non-tumor, chronic autoimmune diseases. We found that CD19-targeted CAR Tregs efficiently suppressed primary human B cells compared with polyclonal Tregs both in vitro and in vivo. Unlike conventional CAR CD8 ${ }^{+} \mathrm{T}$ cells, CAR Tregs may be safer for chronic diseases because CAR Tregs suppressed rather than resulted in GvHD.

Our data are consistent with those of a previous report by Boroughs et al. (43). They also used CD19CAR for modifying costimulatory domains. They concluded that CD19-CAR carrying CD28 signaling domains is superior to 4-1BB-based CD19-CAR in immune suppression, which is consistent with our results. However, there is an important difference, namely, they showed that CD19-targeted CAR Tregs have mild cytotoxicity, while our CD19-CAR Tregs did not. This might be caused by the difference in 
A $\%$ of positive cells
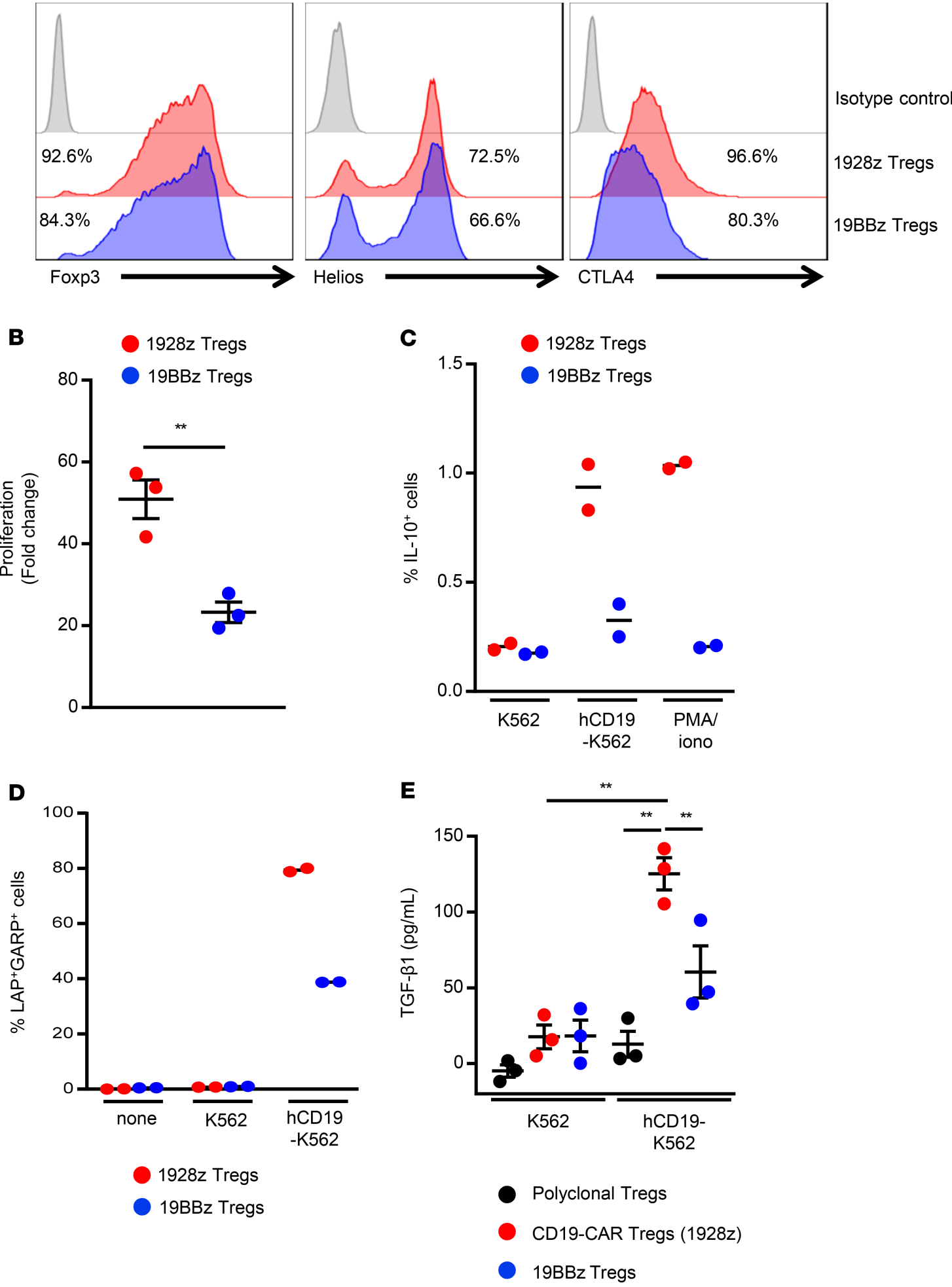

Figure 3. CD28-based CD19-CAR is better than 4-1BB-based CD19-CAR for CAR Treg generation. (A) Flow cytometric analysis of Foxp3, Helios, and CTLA-4 in 1928z Tregs and 19BBz Tregs on day 13. (B) Fold expansion on day 13, 8 days after CAR Tregs were sorted $(n=3)$. (C) Flow cytometric analysis of IL-10 in the indicated cells 4 hours after stimulation with hCD19-K562 cells, K562 cells, or PMA/ionomycin $(n=2)$. (D) Flow cytometric analysis of LAP and GARP in the indicated cells 1 day after coculture with hCD19-K562 or K562 cells $(n=2)$ (E) Amount of TCF- $\beta 1$ produced by CD19-CAR Tregs (1928z) and 19BBz Tregs 1 day after coculture with hCD19-K562 cells or K562 cells $(n=3)$. Data were collected using human samples provided by 1 healthy donor. $P$ values were determined using (B) 2 -tailed Student's $t$ test or $(\mathbf{E}) 1$-way ANOVA $\left({ }^{* *} P<0.01\right)$. Data are presented as mean $\pm \mathrm{SEM}$. 
A Frequency of proliferated B cells CD19-CAR Tregs

Polyclonal Tregs

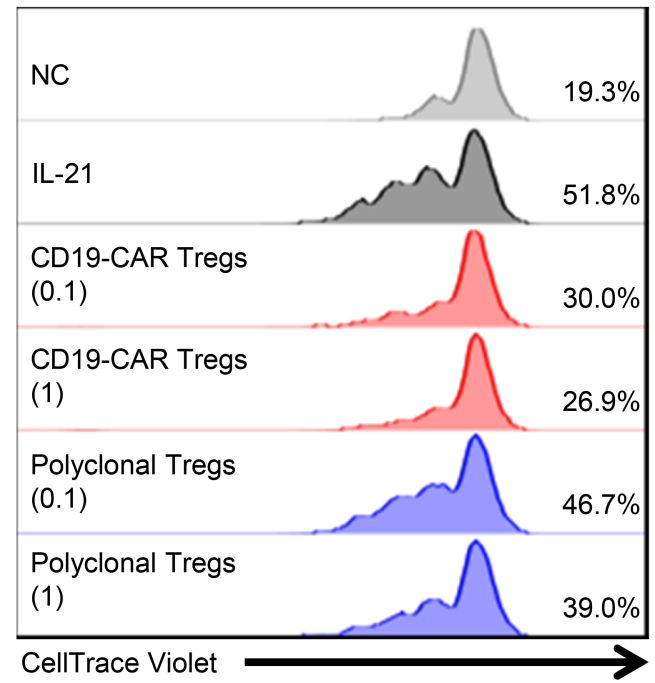

$\mathrm{HD} \# 1$

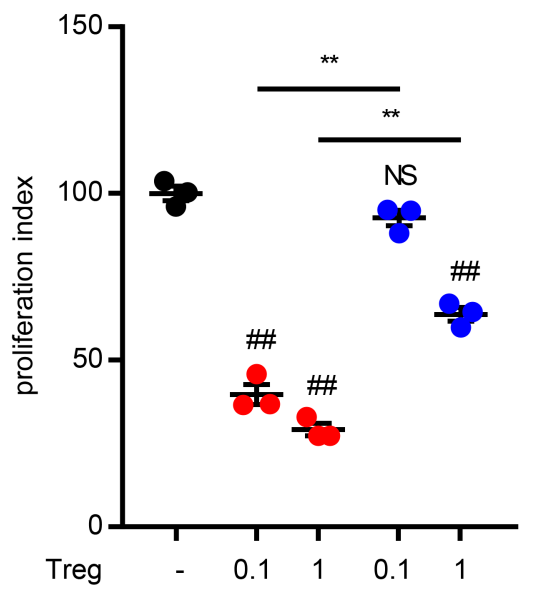

HD \#2

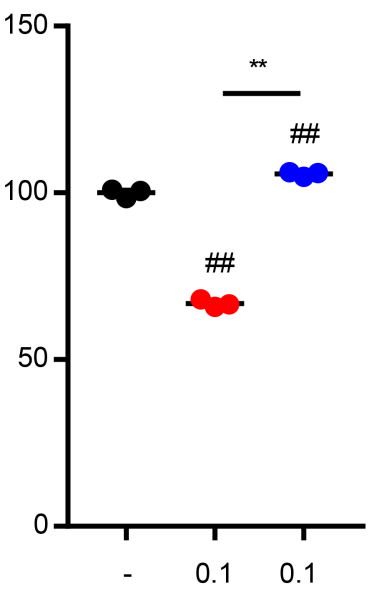

B

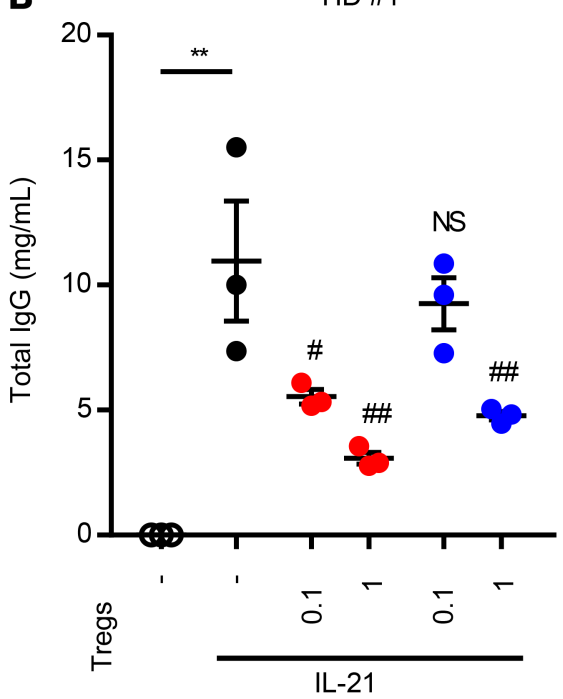

C

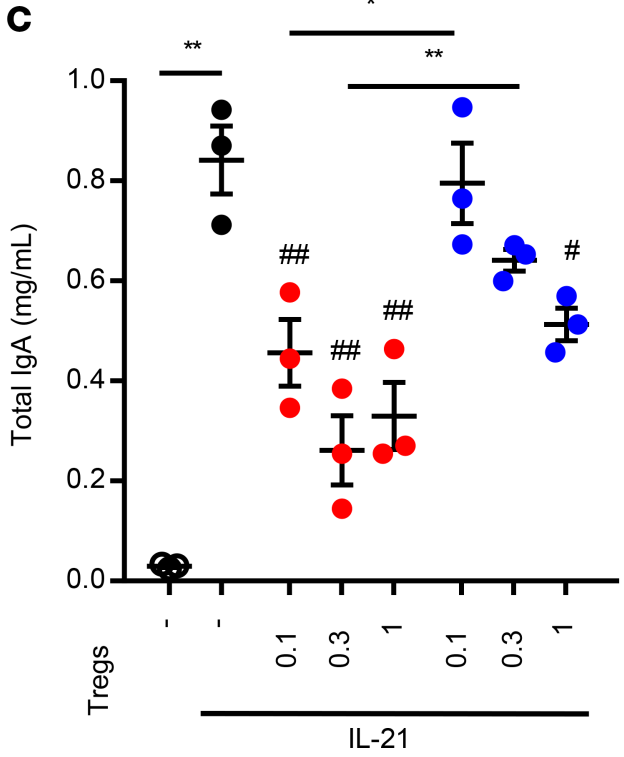

HD \#1

HD \#2
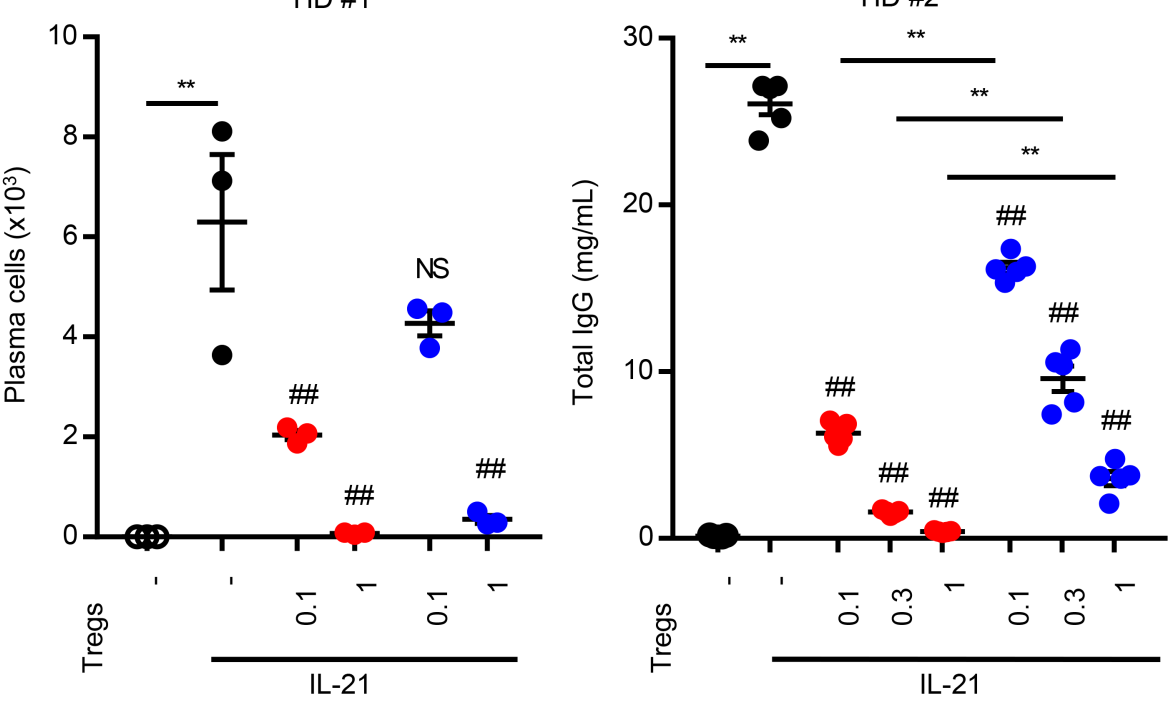

Figure 4. CD19-targeted CAR Tregs efficiently suppress B cells and antibody production. Primary human B cells were stimulated with anti-IgM and anti-CD40 antibodies in the presence of IL-21. (A) Flow cytometric analysis of CellTrace Violet dilution of CellTrace Violet-labeled primary human B cells 3 days after coculture with CD19-CAR Tregs or polyclonal Tregs at ratios of 1:0.1 and 1:1 (B cells/Tregs) $(n=3)$. The fraction of CellTrace Violet ${ }^{\text {to }}$ $B$ cells in the absence of drug and CD19-CAR Tregs is shown as 100\% in the right panels. (B) Total IgG antibody levels produced by B cells and flow cytometric analysis of differentiated B cells (CD4-FVD-IgD-CD38+) 7 days after coculture with CD19-CAR Tregs or polyclonal Tregs at ratios of 1:0.1, 1:0.3, and 1:1 (B cells/Tregs) $(n=3-4)$. HD, healthy donor. (C) Total IgA antibody levels after coculture $(n=3)$. (A and B) Data are representative of independent experiments using samples from 2 healthy donors. (C) Data were collected using human samples provided by 1 healthy donor. (A-C) $P$ values were determined using 1-way ANOVA $\left({ }^{*} P<0.05,{ }^{* *} P<0.01\right.$ compared with the indicated 2 columns; NS, ${ }^{*} P<0.05$, and ${ }^{\# \# P}<0.01$ compared with each black circle). Data are presented as mean \pm SEM. 
A

$\mathrm{HD} \# 1$

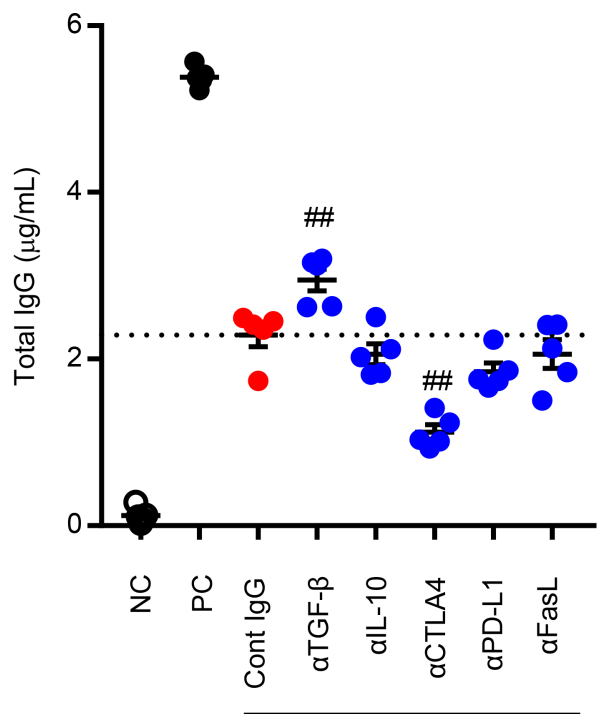

CD19-CAR Tregs

B

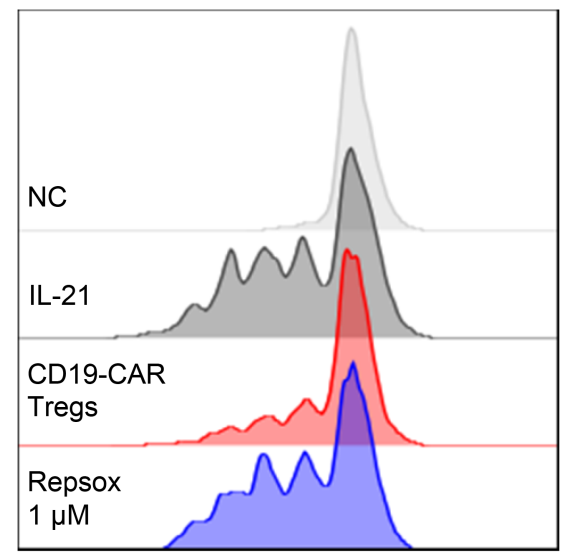

C

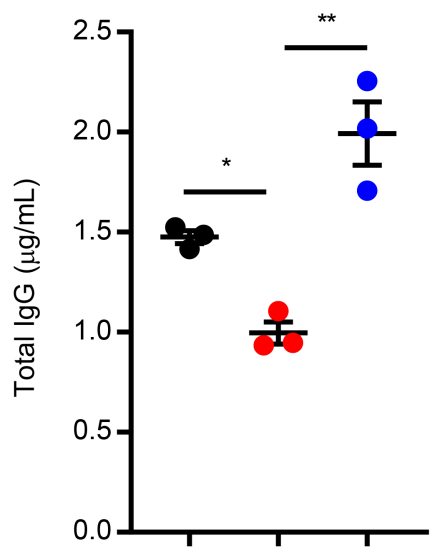

HD \#2
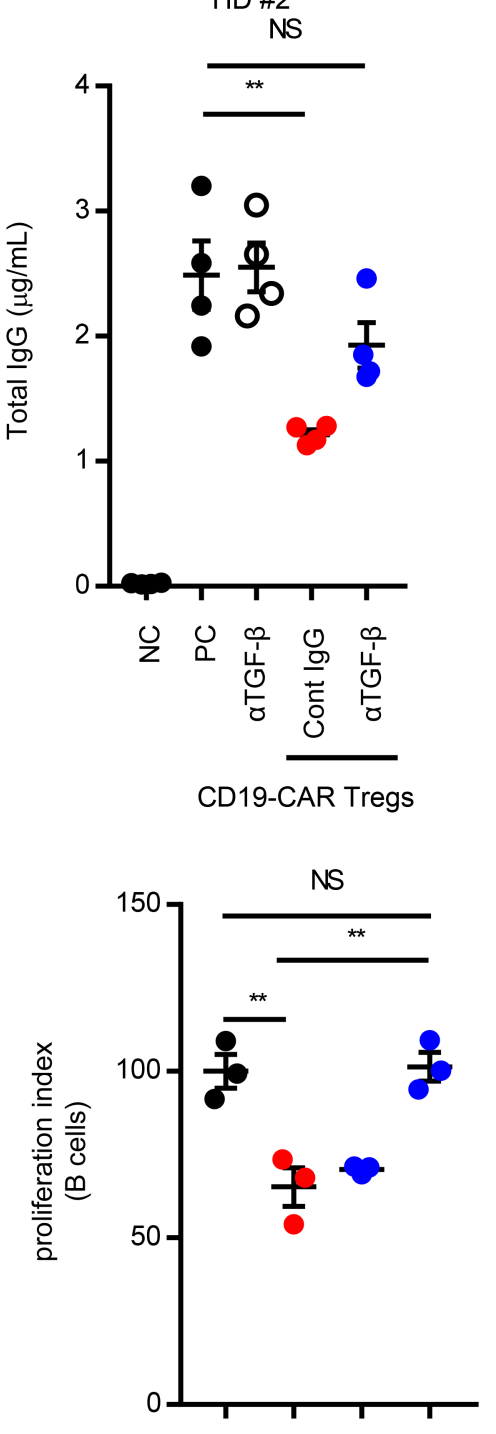

$\operatorname{Repsox}(\mu \mathrm{M}) \quad 0 \quad 0 \quad 0.1 \quad 1$

CD19-CAR Tregs

Figure 5. TGF- $\beta$ from CAR-Tregs play a major role in the suppression of $B$ cell proliferation and IgC production. Primary human $B$ cells were stimulated with anti-IgM and anti-CD40 antibodies in the presence of IL-21. (A) Total IgG antibody levels produced by primary human $B$ cells 7 days after coculture with CD19-CAR Tregs in the presence of various neutralizing antibodies $(10 \mu \mathrm{g} / \mathrm{mL})(n=4-5)$. (B) Flow cytometric analysis of CellTrace Violet dilution of CellTrace Violet-labeled primary human B cells after coculture with CD19-CAR Tregs in the presence of TGF- $\beta$ type 1 receptor inhibitor RepSox ( 0.1 and 1 $\mu \mathrm{M})$. The fraction of CellTrace Violet ${ }^{10} \mathrm{~B}$ cells in the absence of drug and CD19-CAR Tregs is shown as $100 \%$ in the right panel $(n=3)$. NC, negative control; PC, positive control; Cont IgG control IgG. (C) Total IgG antibody levels produced by primary human $B$ cells 7 days after coculture with CD19-CAR Tregs in the presence of RepSox $(1 \mu \mathrm{M})(n=3)$. (A) Data are representative of independent experiments using samples from 2 healthy donors. (B and C) Data were collected using human samples provided by 1 healthy donor. (A-C) $P$ values were determined using 1-way ANOVA ( ${ }^{*} P<0.05$, ${ }^{*} P<0.01$ compared with the indicated 2 columns; NS, \#\# $P<0.01$ compared with each black circle). Data are presented as mean \pm SEM

$\begin{array}{rrrr}\operatorname{Repsox}(\mu \mathrm{M}) & 0 & 0 & 1 \\ \text { CD19-CAR } & - & + & + \\ \text { Tregs } & & & \end{array}$


A

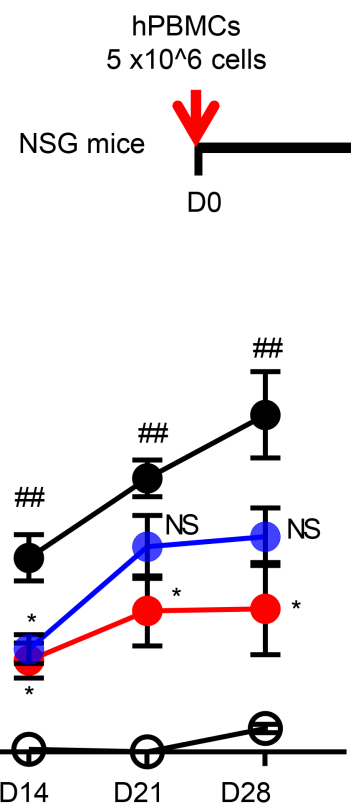

B

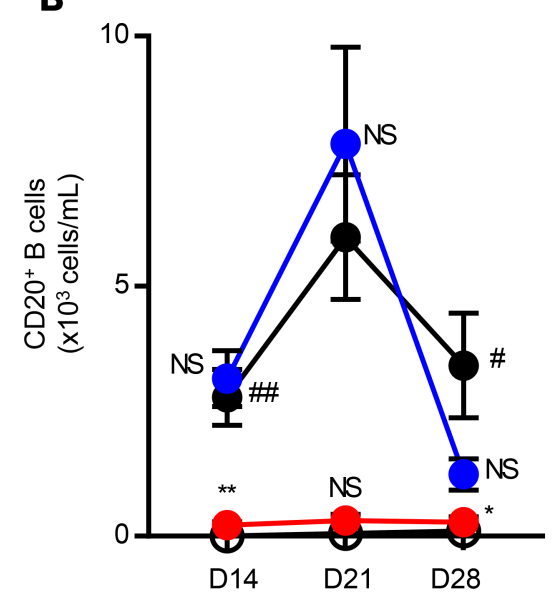

D

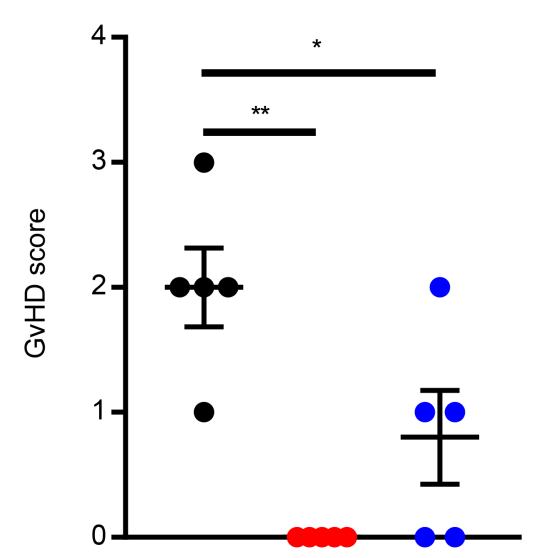

- hPBMCs

CD19-CAR Tregs

Polyclonal Tregs
CD19-CAR Tregs or Polyclonal Tregs $2 \times 10^{\wedge} 6$ cells
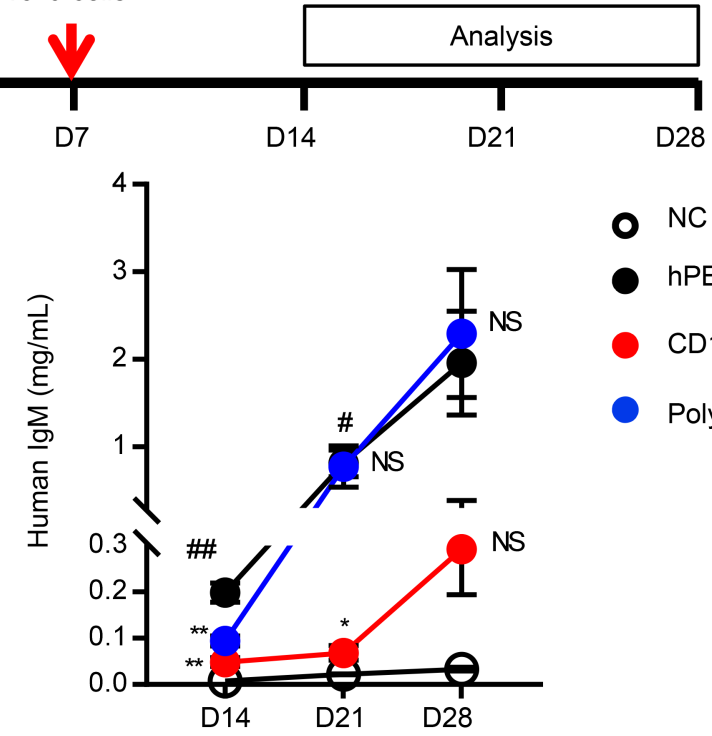

- NC

hPBMCs

CD19-CAR Tregs

Polyclonal Tregs

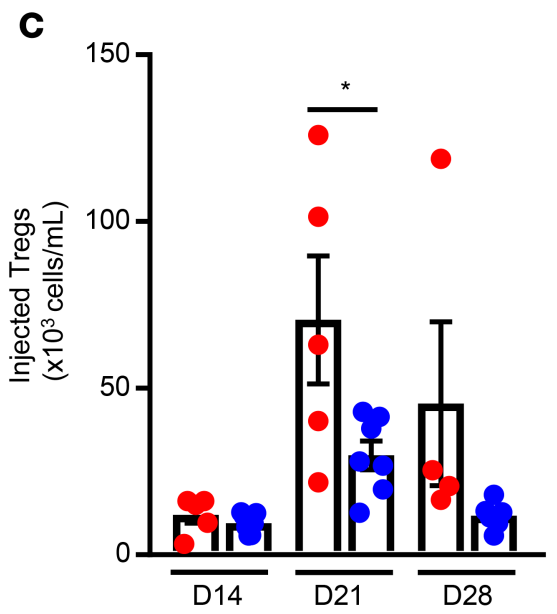

E
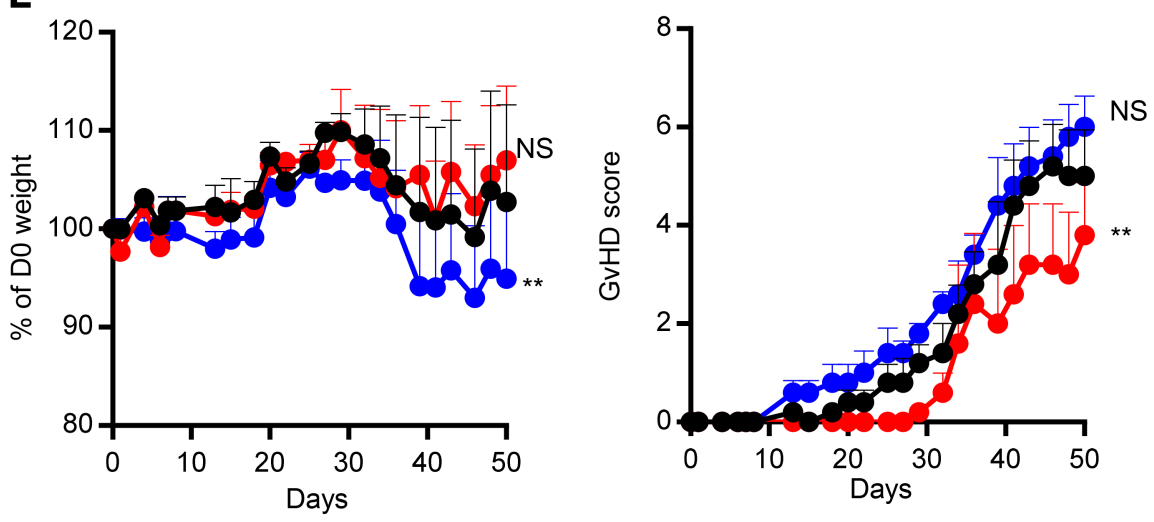

hPBMCs

CD19-CAR Tregs

CD19-CAR CD8 ${ }^{+} T$ cells 
Figure 6. Therapeutic adoptive therapy of CD19-targeted CAR Tregs efficiently suppresses B cells and antibody production in vivo. Severely immunodeficient (NOD.Cg-Prkdc csid/I2rg ${ }^{\text {tmiWjil } / S z ~[N S C]) ~ m i c e ~ w e r e ~ i . v . ~ i n j e c t e d ~ w i t h ~} 5 \times 10^{6}$ human PBMCs. Autologous CD19-CAR Tregs and polyclonal Tregs $\left(2 \times 10^{6}\right)$ were adoptively transferred 7 days after PBMC injection. (A) Total IgG antibody and IgM antibody levels in serum on days 14,21 , and 28 ( $n=4-7)$. (B and C) Number of B cells (FVD-hCD45+hCD20+) (B) and injected Tregs (FVD-hCD45+hCD4+Venus ${ }^{+}$(C) in the peripheral blood on days 14, 21, and 28 measured by flow cytometric analysis $(n=4-7)$. $P$ values was determined using (C) 2 -tailed Student's $t$ test or $(\mathbf{A}, \mathbf{B}$, and $\mathbf{D}) 1$-way ANOVA $\left({ }^{*} P<0.05,{ }^{* *} P<0.01, N S\right.$, comparing red or blue symbols with each black symbol; ${ }^{\#} P<0.05$ and ${ }^{\# \#} P<0.01$, comparing black symbols with each open symbol). Data are presented as the mean \pm SEM. (D) GvHD score was measured on day $28(n=5)$. (E) NSG mice were i.v. injected with $5 \times 10^{6}$ human PBMCs. Autologous CD19-CAR Tregs and CD19-CAR CD8 ${ }^{+}$T cells $\left(2 \times 10^{6}\right)$ were adoptively transferred 7 days after PBMC injection. CvHD score and body weight were measured from day 0 to day $50(n=5)$. (E) $P$ values were determined using 2 -way ANOVA ( ${ }^{*} P<0.05,{ }^{* *} P<0.01$; NS, compared with black circle). Data are presented as mean \pm SEM.

Treg fraction preparation strategy from PBMCs. They used the $\mathrm{CD} 4^{+} \mathrm{CD} 25^{\mathrm{hi}} \mathrm{CD} 127^{\mathrm{lo}}$ fraction as a source of Tregs, whereas we further purified CD45RA ${ }^{+}$cells from this fraction (Figure $1 \mathrm{~A}$ ). The $\mathrm{CD} 4^{+} \mathrm{CD} 25^{\mathrm{hi}} \mathrm{C}-$ D127 fraction contains both naive/resting $\left(C D 45 \mathrm{RA}^{+}\right)$Tregs and CD45RO ${ }^{+}$effector/non-Tregs $(30,31)$. We showed that Foxp3 expression was unstable in $\mathrm{CD}^{2} 5 \mathrm{RO}^{+}$Tregs, and $\mathrm{CD} 45 \mathrm{RO}^{+}$Tregs produced a greater amount of effector cytokines and cytolytic mediators than CD45RA ${ }^{+}$Tregs (Figure 2). Although the fraction of $\mathrm{CD}^{+} \mathrm{CD} 25^{\mathrm{hi}} \mathrm{CD} 127^{10} \mathrm{CD} 45 \mathrm{RA}^{+}$cells was small, we found that it could be expanded vigorously without losing Foxp3 expression and suppression functions; moreover, retroviral gene transduction efficiency in this Treg fraction was higher than in Tconvs, although the exact reason is not clear. Thus, we believe that the source of Tregs is important in order to generate stable and noncytolytic Tregs for Treg therapy.

It has been demonstrated that "exTregs" can be pathogenic and that the stability of Tregs is a bottleneck for Treg therapy (15). In this study, we showed that CD19-CAR CD45RA ${ }^{+}$Tregs did not lose Foxp3 expression after long-term expansion in vitro (Figure 2B); retained Foxp3 expression and persisted for 1 month in NSG mice; and did not exhibit any harmful GvHD effects (Figure 6 and Supplemental Figure 7). Since CD19-CAR CD8 ${ }^{+} \mathrm{T}$ cells persist in the human body in CAR $\mathrm{T}$ therapy, we expect that CD19-CAR Tregs also persist for a long time and maintain Treg functions.

Another proposed CD19-CAR-based cell therapy for autoimmune diseases uses conventional CD19CAR CD8 ${ }^{+} \mathrm{T}$ cells that kill B cells (28). Apparently, CD19-CAR CD8 ${ }^{+} \mathrm{T}$ cells have the potential to induce GvHD or cytokine release syndrome. In contrast, our CD19-CAR CD45RA ${ }^{+}$Tregs suppress B cell proliferation and IgG production but do not kill B cells and have little risk of harmful effects. Anti-CD20 antibody therapy is another way to control B cells in autoimmune diseases (27). Anti-CD20 antibodies also deplete $\mathrm{B}$ cells and cause hypogammaglobulinemia, thereby increasing the risk of infection. CD19-CAR Tregs may also have similar adverse effects; however, we expect that the recovery of Ig after CD19-CAR Treg therapy is faster than after treatment with anti-CD20 depleting antibodies, because CD19-CAR Tregs might just suppress B cells rather than deplete them. To improve the safety of CAR Treg therapy, engineered CARs that are equipped with a suicide gene or work only in the presence of drugs may be made available (18).

In this study, we propose that TGF- $\beta$ is a major mechanism for B cell suppression by CD19-CAR Tregs. Although TGF- $\beta$ is a secreted cytokine, we showed that Treg-B cell direct contact is necessary for the suppression of B cells. This is probably because LAP expression in CD19-CAR Tregs requires the activation of Tregs by target cells. In addition, molecules expressed on $B$ cells such as integrins and proteases may be involved in the processing of LAP to release active TGF- $\beta$ (32). This TGF- $\beta$ activation process may require cell-cell contact, and could be the reason why the anti-TGF- $\beta$ antibody did not revert the effect of CAR Tregs completely, whereas the TGF- $\beta$ signal inhibitor completely reverted it. In addition, TGF- $\beta$-independent mechanisms may also play role especially in vivo; the exact interaction between B cells and CD19CAR Tregs in vivo should be clarified in future studies.

Although our data strongly support that TGF- $\beta$ from CAR Tregs plays a major role in the suppression of B cells, it has not yet been clarified how TGF- $\beta$ signals suppress the proliferation and $\operatorname{IgG}$ production of B cells. Although it is well known that TGF- $\beta$ signals induce IgA class switching $(35,36)$, we have not observed IgA induction by coculture with CD19-CAR Tregs. Exogenous TGF- $\beta$ has been shown to suppress IgG1 or IgG2a production from murine B cells induced by IL-4 or IFN- $\gamma$, respectively (44). Exogenous TGF- $\beta$ has been shown to decrease B lymphocyte Ig secretion by inhibiting the synthesis of Ig mRNA and inhibiting the switch from the membrane form to the secreted forms of Ig mRNAs (45). Further study is necessary to clarify the precise effects of CD19-CAR Tregs on B cells.

CAR T cells could be therapeutic not only for cancer but also for various diseases in which pathogenic cells are involved. A recent article showed that the adoptive transfer of cardiac fibroblast-specific $\mathrm{CD} 8^{+} \mathrm{T}$ cells results in a significant reduction in cardiac fibrosis and restoration of function after injury in mice (46). 
CAR Tregs could be an alternative way to suppress, but not eliminate, pathogenic cells without harmful side effects. The usefulness of CAR-Tregs should be confirmed by using immunocompetent murine models of autoimmunity. However, it is always important to use human $\mathrm{T}$ cells to confirm the therapeutic value of CAR Tregs, because methods for isolation and expansion of pure human Tregs have not been completely established (14).

\section{Methods}

Human subjects. Human PBMCs (hPBMCs) were obtained from 5 healthy human volunteers (male, aged 25-52 years, at Keio University) by density gradient centrifugation.

Cell culture. The K562 cell line (ATCC) was cultured in RPMI-1640 (Nacalai Tesque) supplemented with 10\% FBS (Thermo Fisher Scientific), $10 \mathrm{mM}$ HEPES (Invitrogen), $2 \mathrm{mM}$ L-glutamine (Invitrogen), $1 \times$ NEAA (Invitrogen), $1 \times$ Penicillin-Streptomycin Mixed Solution (Nacalai Tesque), 0.55 mM $\beta$-mercaptoethanol (Thermo Fisher Scientific), and $1 \mathrm{mM}$ sodium pyruvate (Invitrogen). The HEK293T cell line (ATCC) was cultured in DMEM (Nacalai Tesque) supplemented with 10\% FBS and 1× Penicillin-Streptomycin Mixed Solution. Primary human T cells were cultured in RPMI-1640 supplemented with 10\% normal human AB serum (Innovative Research Inc.), 10 mM HEPES, 2 mM L-glutamine, 1× NEAA, 1× Penicillin-Streptomycin Mixed Solution, $0.55 \mathrm{mM} \beta$-mercaptoethanol, and $1 \mathrm{mM}$ sodium pyruvate.

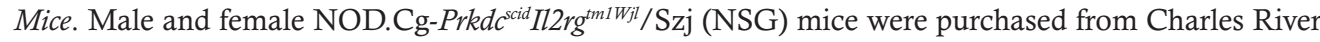
Laboratory Japan. All mice were kept in specific pathogen-free facilities at Keio University.

Plasmid construction and lentiviral transduction. Th human CD19 gene and human HER2 gene were PCR amplified from the human cDNA library, and cloned into lentiviral vector CSII-EF1a-MCS-IRES2-Venus. A method for generating CAR genes was previously described (29). Briefly, CD19-targeted CAR genes - consisting of the signal peptide of human GM-CSFR; anti-human CD19 scFv (FMC63); the extracellular domain, transmembrane region, and cytoplasmic region of human CD28 or human 4-1BB; and

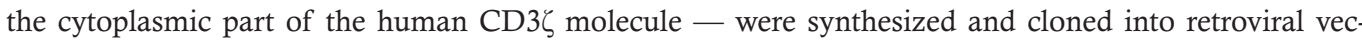
tor pMEI5-containing MCS-IRES2-Venus (pMEI5-MCS-IRES2-Venus). For CD28-based HER2-targeted CAR genes, anti-human HER2 scFv (4D5-7) (38) was used instead of anti-human CD19 scFv (FMC63). The sequences of CARs are described in Supplemental Table 1.

The virus was prepared as previously described (47). Briefly, the cDNA expression vector was transduced into HEK293T cells along with the VSV-G expression vector and packaging vector. Eighteen hours after transduction, the vector-containing culture medium was changed to fresh culture medium; 48 hours later, the virus-containing medium was collected, passed through a $0.45-\mu \mathrm{m}$ filter, and concentrated using centrifugation $\left(8000 \mathrm{~g}\right.$ at $4^{\circ} \mathrm{C}$ for 16 hours). The virus pellets were resuspended in culture medium. K562 cells were transduced to express human CD19 (hCD19-K562) or human HER2 (HER2-K562) with lentivirus.

Flow cytometry. Fluorochrome-conjugated monoclonal/polyclonal anti-human CD4 (45-0049-42, RPA-T4), anti-human CD8 (300924 or 12-0087-41, HIT8 $\alpha$ or SK1), anti-human CD25 (302606, BC96), antihuman CD127 (351316, A019D5), anti-human CD45RA (304126, HI100), anti-human CD45RO (304228, UCHL1), anti-human CD20 (302310 or 13-0209-82, 2H7), anti-human CD45 (45-0459-42, HI30), anti-human CD19 (13-0199-82, HIB19), anti-human CD38 (12-0388-42, HB7), anti-human CD138 (356516, MI15), antihuman IgD (348210, IA6-2), anti-human LAP (349706, TW4-6H10), anti-human GARP (352504, 7B11), anti-human IFN- $\gamma$ (506507, B27), anti-human IL-2 (25-7029-42, MQ1-17H12) anti-human IL-10 (506807, JES3-19F1), propidium iodide (421301; PI), anti-human CTLA-4 (17-1529-42, 14D3), anti-GFP (A-21311, polyclonal), anti-human Foxp3 (17-4777-42, 561182, 236A/E7), anti-human Helios (12-9883-42, 22F6), and fixable viability Dye eFluor 780 (65-0865-14; FVD) were purchased from eBioscience, BioLegend, BD Biosciences, or Thermo Fisher Scientific. Intranuclear staining was performed using fixation/permeabilization buffer solution (eBioscience) or IC fixation buffer (eBioscience), according to the manufacturer's instructions. Stained cells were analyzed on a FACSCanto II (BD Biosciences), and data were analyzed with Diva software (BD Biosciences) and FlowJo software (Tree Star). Flow-count fluorospheres (Beckman Coulter) were used to determine the absolute number of cells, according to the manufacturer's protocol.

Isolation, transduction, and expansion of primary human $T$ cells. $\mathrm{CD}^{+} \mathrm{T}$ cells and $\mathrm{CD} 8^{+} \mathrm{T}$ cells were separated from hPBMCs using a CD4 ${ }^{+} \mathrm{T}$ Cell Isolation Kit, human (Miltenyi Biotec) and a CD8 ${ }^{+} \mathrm{T}$ Cell Isolation Kit, human (Miltenyi Biotec). Naive/resting Tregs $\left(\mathrm{CD}^{+} \mathrm{CD} 25^{+} \mathrm{CD} 127^{-} \mathrm{CD} 45 \mathrm{RA}^{+} \mathrm{CD}^{-} 5 \mathrm{RO}^{-}\right)$ and conventional $\mathrm{CD}^{+} \mathrm{T}$ cells (Tconvs; $\mathrm{CD} 4^{+} \mathrm{CD} 25^{-} \mathrm{CD} 127^{+} \mathrm{CD} 45 \mathrm{RA}^{+} \mathrm{CD} 45 \mathrm{RO}^{-}$) were isolated from 
MACS-purified CD4 ${ }^{+} \mathrm{T}$ cells by FACS using an Aria II/III cell sorter (BD Biosciences). Naive/resting Tregs were stimulated with anti-CD3/CD28 Dynabeads (Life Technologies) in the presence of $100 \mathrm{ng} /$ mL human IL-2 (PeproTech). On day 2, CD19-targeted CAR gene or HER2-targeted CAR gene (described above) was transduced with retrovirus by centrifugation at $35^{\circ} \mathrm{C}$ for 2 hours in the presence of $5 \mu \mathrm{g} / \mathrm{mL}$ polybrene (Nacalai Tesque). On day 5 or 6 , Venus $^{+}$cells were isolated by a FACSAria II/III cell sorter and expanded by coculture with irradiated (40 Gy) antigen-expressing K562 cells (CD19-K562 cells for CD19-targeted CAR and HER2-K562 cells for HER2-targeted CAR) in the presence of $100 \mathrm{ng} / \mathrm{mL}$ human IL-2. Other irradiated antigen-expressing K562 cells were added 3 or 4 days after the first coculture. Polyclonal Tregs that were transduced with an empty gene or not transduced were cultured with anti-CD3/ CD28 Dynabeads in the presence of $100 \mathrm{ng} / \mathrm{mL}$ human IL-2. Tconvs and CD8 ${ }^{+} \mathrm{T}$ cells were cultured in the presence of 10 and $30 \mathrm{ng} / \mathrm{mL}$ human IL-2, respectively, and gene transduction was carried out on day 1. All T cells were cultured at $4 \times 10^{4}$ to $10 \times 10^{4}$ cells/well in 96-well round plates after gene transduction. For purification after expansion, CAR $\mathrm{T}$ cells were stained with biotin-conjugated anti-human CD235a antibody (13-9987-82, HIR2, eBioscience), followed by incubation with anti-biotin microbeads (Miltenyi Biotec). CAR T cells were then negatively selected by MACS.

Cytotoxicity assays and cytokine production. T cells were cocultured with irradiated (40 Gy) CD19-K562 cells (labeled with CellTrace Violet [Thermo Fisher Scientific] in PBS at $37^{\circ} \mathrm{C}$ for 8 minutes) at a ratio of 4:1 or 1:1 ( $\mathrm{T}$ cells/targeted cells) for 16-20 hours. The culture supernatants were then collected, and the percentage of dead cells in the targeted cells $\left(\mathrm{PI}^{+}\right.$in CellTrace Violet ${ }^{+}$) or surface TGF- $\beta$ expression $\left(\mathrm{LAP}^{+} \mathrm{GARP}^{+}\right)$in $\mathrm{CD}^{+} \mathrm{T}$ cells was estimated using flow cytometry analysis. Concentrations of cytokines (TGF- $\beta 1$, IFN- $\gamma$, and IL-2) in the culture supernatants were measured using ELISA (eBioscience). For intracellular cytokine staining, CAR T cells were stimulated with irradiated (40 Gy) CD19-K562 cells and K562 cells at a 1:1 ratio, or with PMA (50 ng/mL, Nacalai Tesque)/ionomycin ( $1 \mu \mathrm{g} / \mathrm{mL}$, Nacalai Tesque) for 4-5 hours in the presence of Brefeldin A (1:1000 dilution, eBioscience). Then, intracellular IFN- $\gamma$, IL-2, and IL-10 were stained and analyzed by flow cytometry, as previously described (48).

$B$ cell suppression assay and antibody production assay in vitro. Primary human B cells were isolated from hPBMCs using a B Cell Isolation Kit II, human (Miltenyi Biotec) and stimulated with anti-IgM (Jackson ImmunoResearch Laboratories Inc.) and anti-CD40 antibodies (R\&D Systems) in the presence of $100 \mathrm{ng} /$ mL IL-21 (PeproTech) for 3 days (suppression assay) and 7 days (antibody production assay) $\left(2 \times 10^{4}\right.$ to $5 \times$ $10^{4}$ cells/well in 96-well round plates) (34). In some experiments, we added RepSox (MilliporeSigma, 1 or 10 $\mu \mathrm{M})$ or neutralizing antibody $(10 \mu \mathrm{g} / \mathrm{mL})$; anti-TGF- $\beta$ antibody (16-9243-85, 1D11.16.8, eBioscience), antiIL-10 antibody (16-7108-85, JES3-9D7, eBioscience), anti-CTLA-4 antibody (16-1529-82, 14D3, eBioscience), anti-PD-L1 antibody (16-5983-82, MIH1, eBioscience), and anti-FasL antibody (16-9919-81, NOK-1, eBioscience). In the suppression assay, B cells were labeled with CellTrace Violet in PBS at $37^{\circ} \mathrm{C}$ for 8 minutes. CD19-targeted CAR Tregs (CD19-CAR Tregs) or empty/polyclonal Tregs (polyclonal Tregs) were cocultured with $B$ cells at a ratio of 1:1/10, 1:3/10, or 1:1 (B cells/Tregs). In a suppression assay, proliferated $\mathrm{B}$ cells $\left(\mathrm{CD} 4^{-}\right.$CellTrace Violet $^{10}$ ) were analyzed by flow cytometry. In an antibody production assay, differentiated B cells (FVD $\left.{ }^{-} \mathrm{CD}^{-} \mathrm{IgD}^{-} \mathrm{CD} 38^{+}\right)$were estimated by flow cytometry, and the concentration of human total IgG antibody and IgA antibody in the supernatant was measured by ELISA (Bethyl Laboratories). In some experiments, Transwell plates $(0.4 \mu \mathrm{m}$; Corning) were used to investigate the contact-dependent mechanism. "Proliferation index" was calculated as the percentage of divided B cells (CellTrace Violet ${ }^{l o}$ fraction).

CD19-targeted CAR Tconv suppression assay. CD19-targeted CAR Tconvs (CD19-CAR Tconvs) on day 13 were labeled with CellTrace Violet in PBS at $37^{\circ} \mathrm{C}$ for 8 minutes and stimulated with anti-CD3/CD28 Dynabeads (cells/beads, 10:1) for 2 days. CD19-targeted CAR Tregs (CD19-CAR Tregs) or empty vectortransduced Tregs (polyclonal Tregs) were cocultured with CD19-CAR Tconvs at a ratio of 1:1 (Tconvs/ Tregs). The number of CD19-CAR Tconvs $\left(C D 4^{+}\right.$CellTrace Violet $\left.{ }^{+}\right)$was analyzed by flow cytometry.

Xenograft mouse model for antibody production in vivo. Eight- to 11-week-old male and female NSG mice were i.v. injected with $2 \times 10^{6}$ CD19-targeted CAR Tregs or empty Tregs 4-6 hours or 7 days after i.v. injection of $5 \times 10^{6} \mathrm{hPBMCs}$. Saline-injected mice served as controls. On days 14, 21, and 28, peripheral blood and spleens were collected, and erythrocytes were lysed. B cells $\left(\mathrm{FVD}^{-} \mathrm{hCD} 45^{+} \mathrm{hCD} 20^{+}\right)$, inject-

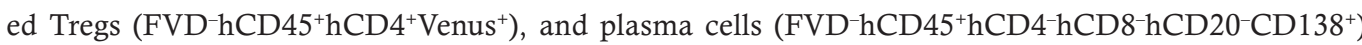
were measured by flow cytometry. Serum concentrations of human total IgG antibody and IgM antibody were measured by ELISA (Bethyl Laboratories). To estimate GvHD, body weight, GvHD score, and survival were measured from day 0 to day $>100$. GvHD score was calculated based on body weight, posture, 
activity level, fur texture, and skin integrity, with 0-2 points per category, as previously described (49). Mice were euthanized if they experienced a GvHD score greater than 8. In some experiments, hPBMCs were stained with biotin-conjugated anti-human CD19/CD20 antibody, followed by incubation with anti-biotin microbeads, and then B cell-deficient hPBMCs were negatively selected by MACS. B cell-sufficient or B cell-deficient hPBMCs $\left(5 \times 10^{6}\right)$ were i.v. injected into NSG mice.

Statistics. All data are presented as mean \pm SEM. Statistical analyses were performed using Student's unpaired $t$ test (2-tailed) or 1-or 2-way ANOVA with Tukey's tests, using GraphPad Prism version 7 software (GraphPad Software). $P<0.05$ was considered to be statistically significant.

Study approval. Animal experiments were performed in strict accordance with the recommendations in the Guidelines for Proper Conduct of Animal Experiments of the Science Council of Japan. All experiments using mice were approved by the Animal Ethics Committee of Keio University (approval 08004) and were performed according to the Animal Ethics Committee's guidelines. All human study was approved by the Institutional Review Board of Keio University School of Medicine (approval 20120039), and written informed consent was obtained from all 5 participants.

\section{Author contributions}

YI, MI, TK, and MA performed the experiments; YI analyzed the data; AY supervised the research; and YI, MA, and AY wrote the manuscript.

\section{Acknowledgments}

We thank Mari Ikeda, Yoshiko Noguchi, Yasuko Hirata, and Yukiko Tokifuji (Keio University) for technical assistance; Mika Inoue (Keio University) and Kasane Imura-Kishi (University of Tokyo) for manuscript preparation; and Yuzo Koda (Keio University) for discussions. This work was supported by Japan Society for the Promotion of Science (JSPS) KAKENHI (S) JP17H06175, Challenging Research (P) JP18H05376, and AMED-CREST JP19gm1110009 grants to AY; and JSPS KAKENHI 17K15667, 19H04817, and 19K16618, AMED-PRIME 19gm6210012 to MI; and by the Tomizawa Jun-ichi \& Keiko Fund of the Molecular Biology Society of Japan for Young Scientists, a research grant for young investigators from the Mitsubishi Foundation, Mochida Memorial Foundation for Medical and Pharmaceutical Research, Takeda Science Foundation, Uehara Memorial Foundation, Naito Memorial Foundation, Kanae Foundation, SENSHIN Medical Research Foundation, Astellas Foundation for Research on Metabolic Disorders, Inoue Research Award, Life Science Research Award, the Princess Takamatsu Cancer Research Fund, a Bristol-Myers Squibb Research Grant, and Keio Gijuku Academic Developmental Funds.

Address correspondence to: Akihiko Yoshimura or Yuki Imura, Department of Microbiology and Immunology, Keio University School of Medicine, 35 Shinanomachi, Shinjuku-ku, Tokyo 160-8582, Japan. Phone: 81.3.5363.3483; Email: yoshimura@keio.jp (A. Yoshimura) or imura.yuuki@ma.mt-pharma.co.jp (Y. Imura).

1. Sakaguchi S, Yamaguchi T, Nomura T, Ono M. Regulatory T cells and immune tolerance. Cell. 2008;133(5):775-787.

2. Brunstein CG, et al. Infusion of ex vivo expanded T regulatory cells in adults transplanted with umbilical cord blood: safety profile and detection kinetics. Blood. 2011;117(3):1061-1070.

3. Di Ianni M, et al. Tregs prevent GVHD and promote immune reconstitution in HLA-haploidentical transplantation. Blood. 2011;117(14):3921-3928.

4. Theil A, et al. Adoptive transfer of allogeneic regulatory T cells into patients with chronic graft-versus-host disease. Cytotherapy. 2015;17(4):473-486

5. Trzonkowski $P$, et al. First-in-man clinical results of the treatment of patients with graft versus host disease with human ex vivo expanded CD4+CD25+CD127- T regulatory cells. Clin Immunol. 2009;133(1):22-26.

6. Bluestone JA, et al. Type 1 diabetes immunotherapy using polyclonal regulatory T cells. Sci Transl Med. 2015;7(315):315ra189.

7. Marek-Trzonkowska N, et al. Therapy of type 1 diabetes with CD4(+)CD25(high)CD127-regulatory T cells prolongs survival of pancreatic islets - results of one year follow-up. Clin Immunol. 2014;153(1):23-30.

8. Marek-Trzonkowska N, et al. Administration of CD4+CD25highCD127-regulatory T cells preserves $\beta$-cell function in type 1 diabetes in children. Diabetes Care. 2012;35(9):1817-1820.

9. Adair PR, Kim YC, Zhang AH, Yoon J, Scott DW. Human Tregs made antigen specific by gene modification: the power to treat autoimmunity and antidrug antibodies with precision. Front Immunol. 2017;8:1117.

10. Dawson NAJ, Vent-Schmidt J, Levings MK. Engineered tolerance: tailoring development, function, and antigen-specificity of regulatory T cells. Front Immunol. 2017;8:1460.

11. Sagoo P, Ali N, Garg G, Nestle FO, Lechler RI, Lombardi G. Human regulatory T cells with alloantigen specificity are more 
potent inhibitors of alloimmune skin graft damage than polyclonal regulatory T cells. Sci Transl Med. 2011;3(83):83ra42.

12. Kim YC, et al. Engineered antigen-specific human regulatory T cells: immunosuppression of FVIII-specific T-and B-cell responses. Blood. 2015;125(7):1107-1115.

13. Kim YC, et al. Engineered MBP-specific human Tregs ameliorate MOG-induced EAE through IL-2-triggered inhibition of effector T cells. J Autoimmun. 2018;92:77-86.

14. Kasahara H, et al. Generation of allo-antigen-specific induced Treg stabilized by vitamin $\mathrm{C}$ treatment and its application for prevention of acute graft versus host disease model. Int Immunol. 2017;29(10):457-469.

15. Kanamori M, Nakatsukasa H, Okada M, Lu Q, Yoshimura A. Induced regulatory t cells: their development, stability, and applications. Trends Immunol. 2016;37(11):803-811.

16. Chavez JC, Bachmeier C, Kharfan-Dabaja MA. CAR T-cell therapy for B-cell lymphomas: clinical trial results of available products. Ther Adv Hematol. 2019;10:2040620719841581.

17. Mikkilineni L, Kochenderfer JN. Chimeric antigen receptor T-cell therapies for multiple myeloma. Blood. 2017;130(24):2594-2602.

18. Hartmann J, Schüßler-Lenz M, Bondanza A, Buchholz CJ. Clinical development of CAR T cells-challenges and opportunities in translating innovative treatment concepts. EMBO Mol Med. 2017;9(9):1183-1197.

19. MacDonald KG, et al. Alloantigen-specific regulatory T cells generated with a chimeric antigen receptor. J Clin Invest. 2016;126(4):1413-1424.

20. Yoon J, Schmidt A, Zhang AH, Königs C, Kim YC, Scott DW. FVIII-specific human chimeric antigen receptor T-regulatory cells suppress T- and B-cell responses to FVIII. Blood. 2017;129(2):238-245.

21. Fransson M, et al. CAR/FoxP3-engineered T regulatory cells target the CNS and suppress EAE upon intranasal delivery. $J$ Neuroinflammation. 2012;9:112.

22. Elinav E, Adam N, Waks T, Eshhar Z. Amelioration of colitis by genetically engineered murine regulatory T cells redirected by antigen-specific chimeric receptor. Gastroenterology. 2009;136(5):1721-1731.

23. Rawlings DJ, Metzler G, Wray-Dutra M, Jackson SW. Altered B cell signalling in autoimmunity. Nat Rev Immunol. 2017;17(7):421-436.

24. Murphy G, Isenberg DA. New therapies for systemic lupus erythematosus - past imperfect, future tense. Nat Rev Rheumatol. 2019;15(7):403-412.

25. Nocturne G, Mariette X. B cells in the pathogenesis of primary Sjögren syndrome. Nat Rev Rheumatol. 2018;14(3):133-145.

26. Watanabe T, Minaga K, Kamata K, Kudo M, Strober W. Mechanistic insights into autoimmune pancreatitis and IgG4-related disease. Trends Immunol. 2018;39(11):874-889.

27. Rubin SJS, Bloom MS, Robinson WH. B cell checkpoints in autoimmune rheumatic diseases. Nat Rev Rheumatol. 2019;15(5):303-315.

28. Kansal R, et al. Sustained B cell depletion by CD19-targeted CAR T cells is a highly effective treatment for murine lupus. Sci Transl Med. 2019;11(482):eaav1648.

29. Kondo T, et al. The NOTCH-FOXM1 axis plays a key role in mitochondrial biogenesis in the induction of human stem cell memory-like CAR-T cells. Cancer Res. 2020;80(3):471-483.

30. Wing JB, Tanaka A, Sakaguchi S. Human FOXP3 ${ }^{+}$regulatory $\mathrm{T}$ cell heterogeneity and function in autoimmunity and cancer. Immunity. 2019;50(2):302-316.

31. Miyara M, et al. Functional delineation and differentiation dynamics of human CD4+ T cells expressing the FoxP3 transcription factor. Immunity. 2009;30(6):899-911.

32. Yoshimura A, Muto G. TGF- $\beta$ function in immune suppression. Curr Top Microbiol Immunol. 2011;350:127-147.

33. Shevach EM. Garp as a therapeutic target for modulation of T regulatory cell function. Expert Opin Ther Targets. 2017;21(2):191-200.

34. Ettinger R, et al. IL-21 induces differentiation of human naive and memory B cells into antibody-secreting plasma cells. $J$ Immunol. 2005;175(12):7867-7879.

35. Coffman RL, Lebman DA, Shrader B. Transforming growth factor beta specifically enhances IgA production by lipopolysaccharide-stimulated murine B lymphocytes. J Exp Med. 1989;170(3):1039-1044.

36. van Vlasselaer P, Punnonen J, de Vries JE. Transforming growth factor-beta directs IgA switching in human B cells. J Immunol. 1992;148(7):2062-2067.

37. Long AH, et al. 4-1BB costimulation ameliorates $\mathrm{T}$ cell exhaustion induced by tonic signaling of chimeric antigen receptors. Nat Med. 2015;21(6):581-590.

38. Liu X, et al. Affinity-tuned ErbB2 or EGFR chimeric antigen receptor T Cells exhibit an increased therapeutic index against tumors in mice. Cancer Res. 2015;75(17):3596-3607.

39. Tsuchida Y, et al. TGF-B3 Inhibits antibody production by human B Cells. PLoS One. 2017;12(1):e0169646.

40. Søndergaard H, Kvist PH, Haase C. Human T cells depend on functional calcineurin, tumour necrosis factor- $\alpha$ and CD80/ CD86 for expansion and activation in mice. Clin Exp Immunol. 2013;172(2):300-310.

41. Davila ML, et al. Efficacy and toxicity management of 19-28z CAR T cell therapy in B cell acute lymphoblastic leukemia. Sci Transl Med. 2014;6(224):224ra25.

42. Barrett DM, Teachey DT, Grupp SA. Toxicity management for patients receiving novel T-cell engaging therapies. Curr Opin Pediatr. 2014;26(1):43-49.

43. Boroughs AC, et al. Chimeric antigen receptor costimulation domains modulate human regulatory T cell function. JCI Insight. 2019;4(8):e126194.

44. Garcia B, Rodríguez R, Angulo I, Heath AW, Howard MC, Subiza JL. Differential effects of transforming growth factor-beta 1 on IgA vs. IgG2b production by lipopolysaccharide-stimulated lymph node B cells: a comparative study with spleen B cells. Eur J Immunol. 1996;26(10):2364-2370.

45. Kehrl JH, Thevenin C, Rieckmann P, Fauci AS. Transforming growth factor-beta suppresses human B lymphocyte Ig production by inhibiting synthesis and the switch from the membrane form to the secreted form of Ig mRNA. J Immunol. 1991;146(11):4016-4023.

46. Aghajanian H, et al. Targeting cardiac fibrosis with engineered T cells. Nature. 2019;573(7774):430-433.

47. Ichiyama K, et al. Transcription factor Smad-independent T helper 17 cell induction by transforming-growth factor- $\beta$ is mediat- 
ed by suppression of eomesodermin. Immunity. 2011;34(5):741-754.

48. Kondo T, et al. Notch-mediated conversion of activated $\mathrm{T}$ cells into stem cell memory-like $\mathrm{T}$ cells for adoptive immunotherapy. Nat Commun. 2017;8:15338.

49. Cooke KR, et al. An experimental model of idiopathic pneumonia syndrome after bone marrow transplantation: I. The roles of minor H antigens and endotoxin. Blood. 1996;88(8):3230-3239. 